Research Article

\title{
Robust Model Predictive Control for Energy Management of Isolated Microgrids Based on Interval Prediction
}

\author{
Huihui He $\mathbb{D}^{1,2}$ Shengjun Huang $\mathbb{D}^{1,2}$ Yajie Liu $\left.\mathbb{D}\right)^{1,2}$ and Tao Zhang $\mathbb{D}^{1,2}$ \\ ${ }^{1}$ College of Systems Engineering, National University of Defense Technology, Changsha 410073, Hunan, China \\ ${ }^{2}$ Hunan Key Laboratory of Multi-Energy System Intelligent Interconnection Technology (HKL-MSI2T), Changsha 410073, \\ Hunan, China
}

Correspondence should be addressed to Huihui He; hehuihui@nudt.edu.cn

Received 18 September 2021; Accepted 30 September 2021; Published 14 October 2021

Academic Editor: Shi Cheng

Copyright (C) 2021 Huihui He et al. This is an open access article distributed under the Creative Commons Attribution License, which permits unrestricted use, distribution, and reproduction in any medium, provided the original work is properly cited.

With the integration of Renewable Energy Resources (RERs), the Day-Ahead (DA) scheduling for the optimal operation of the integrated Isolated Microgrids (IMGs) may not be economically optimal in real time due to the prediction errors of multiple uncertainty sources. To compensate for prediction error, this paper proposes a Robust Model Predictive Control (RMPC) based on an interval prediction approach to optimize the real-time operation of the IMGs, which diminishes the influence from prediction error. The rolling optimization model in RMPC is formulated into the robust model to schedule operation with the consideration of the price of robustness. In addition, an Online Learning (OL) method for interval prediction is utilized in RMPC to predict the future information of the uncertainties of RERs and load, thereby limiting the uncertainty. A case study demonstrates the effectiveness of the proposed with the better matching between demand and supply compared with the traditional Model Predictive Control (MPC) method and Hard Charging (HC) method.

\section{Introduction}

As smart parts of the future power grid, the microgrid is an active distribution system integrated with Distributed Generators (DGs), Energy Storage Systems (ESS), controllable loads, and other electric components [1]. It can operate in either grid-connected mode or islanded mode and the energy schedule can be addressed by an energy management system (EMS). Besides, with the increasing penetration of Renewable Energy Resources (RERs), microgrids have developed the ability to confirm power supply prosperity and enhance energy efficiency. However, the uncertainty of RERs also brings great challenges to EMS for Islanded Microgrids (IMGs) to balance the demand and supply without the violation of the operating constraints. Additionally, there are usually larger prediction errors in RERs compared to the rest of the uncertainties causing a large impact on energy management [2]. Therefore, this paper focuses on the energy management in IMGs to reduce the operation cost with the consideration of the uncertainties in power generation of RERs and the demand level of the user loads.

Energy management takes into account strategic decisions related to the status and the generated power of DGs and operation decisions regarding the charging/discharging of ESS, the ratio of load shedding on the cutting load, and the power flow through the transmission to meet electricity demands at an acceptable economic cost [3]. However, this problem has become more complex under combined demand and supply uncertainty. In the literature, several previous studies have incorporated various uncertainties in the energy management problem, aiming to optimize the economic cost and the environmental cost. Wang et al. [4] established a multiobjective operation optimization model taking the economic benefits and comprehensive energy efficiency as the objective function based on a novel demand response mechanism. Ji et al. [5] proposed a mixed-integer linear programming optimization model with the aim of minimum total annualized cost for a stand-alone hybrid energy system with PV and biomass-CHP. Lorestani et al. 
[6] introduced a rule-based energy management system to administrate the stand-alone multicarrier microgrid operation and maximize RERs utilization. Peláez-Peláez et al. [7] proposed a techno-economic assessment for a hybrid combined generation system based on renewable storage technologies. Maulik and Das [8] presented a method for determining optimal droop settings of dispatchable distributed generation units in an IMG aiming at minimizing the operational cost and the emission with the consideration of the uncertainties of load and renewable generation forecasting. Wang et al. [9] provided a novel microgrid planning methodology to decide optimal locations, sizes, and mix of dispatchable and intermittent DGs to reduce the long-term costs.

During the past decades, Model Predictive Control (MPC) has been well developed for power system and power plant control due to the advantage of systematic handling of the uncertainties of the RERs in a forward-looking way when optimizing the hourly schedule $[10,11]$. Parisio et al. [12] presented a study on applying an MPC approach to an experimental microgrid located in Athens, Greece, to assess the performance of the online optimization-based control strategy. Turk et al. [13] proposed an MPC-based real-time scheduling strategy to optimize the real-time operation of the integrated energy system based on the measured state of the system and future information of uncertainties. Ye et al. [14] presented a novel Hierarchical Model Control (HMPC) strategy based on dynamic active power dispatch to improve wind power schedule and increase wind power accommodation, including intraday dispatch, real-time dispatch, cluster optimization, and wind farm modulation layer. Yin and Su [15] provided a multistep depth Model Predictive Control algorithm based on maximum power point tracking techniques to increase the PV power generation efficiency by combining deep neural networks and Model Predictive Control. Hu et al. [16] proposed a sophisticated control algorithm consisting of a Model Predictive Voltage Control (MPVC) and a Model Predictive Power Control (MPPC) for the interlinking converter to control the battery energy storage (BES) to smooth the PV output. Shi et al. [17] developed a novel Alternating Direction Method of Multipliers- (ADMM-) based distributed MPC algorithm for the online scheduling problem of the joint coordination of demand response and AC optimal power flow with curtailment of RERs to save the cost and manage the fluctuation of the overall power load.

Similar to $[13,14]$, the optimal operation planning of the IMGs is tackled with the consideration of the uncertainty of RERs and load demand by the MPC method. Moreover, we make the following three improvements: with respect to the model, we formulate the energy management of IMGs problem into a robust model with the definition of the price of robustness, which releases the impact from the uncertainty [18]. For the method, we develop a Robust Model Prediction Control (RMPC) approach based on interval prediction to generate optimal solutions aiming at achieving higher flexibility and economic efficiency. Besides, an Online Learning (OL) method is applicable for more accurate predicted results in interval prediction.
The main contributions of this paper can be summarized as follows:

(i) With the consideration of uncertainties of RERs and load demand, this paper proposes an RMPC method for the energy management of IMGs based on interval prediction to reduce operational costs and improve the utilization of RERs. The price of robustness is implemented to present the preference of the planner about the risk of power shortage.

(ii) Multiple uncertainty sources are taken into account for the real-time (RT) scheduling of the IMGs, including the load demand and the outputs of RERs. The OL application combined with RMPC is used to provide time-efficient and accurate interval predictions one step ahead for the RMPC to reduce future forecast error.

(iii) Case studies are implemented to investigate the effectiveness of the RMPC method based on the interval prediction for management under uncertainties. The performance of the proposed method is compared with the conventional MPC method and Hard Charging (HC) strategy approach for the power system. Moreover, the economic efficiency of the prediction horizon length of the RMPC is also discussed in this paper.

The rest of this paper is organized as follows. Section 2 presents the formulation of the energy management model of IMGs and the uncertain set of RERs and load demand for the proposed problem. In Section 3, an overview of the traditional MPC for real-time scheduling and the framework of RMPC based on interval prediction are given. Numerical experiments are implemented in Section 4. Finally, conclusions and future research are given in Section 5.

\section{Problem Formulation and the Proposed Model}

In this section, the mathematical formulation and the proposed optimization of the isolated MG for a Day-Ahead (DA) time horizon planning are presented. The IMGs system consists of controllable loads, ESS, DGs, and RERs, such as wind turbines and photovoltaic (PV) arrays.

2.1. Mathematical Formulation. The RMPC-based energy management aims to find an optimal solution depending on the updated measured state and future interval predictions in the specified prediction horizon. The objective is conducted by equation (1) for minimizing the operation cost including the DG costs, the ESS costs, and the penalty of load shedding. The DG costs consist of the fixed cost of starting up/shutting down and maintaining a potential DG unit (the first and the second term in the corresponding equation (3)) and the variable cost of DGs operating depending on the power output under each period (the third term). The ESS costs include the cost of maintaining ESS according to the charging/discharging power transmitted in the IMG. The penalty of load shedding is accounted for in the third part in 
equations (1) and (7). It should be mentioned that the load demand of the critical facilities must be satisfied so it is feasible to cut the proportional energy supply to meet the need of the critical loads. The mathematical formulation of RMPC for energy management is introduced as follows:

$$
\begin{aligned}
C_{\text {total }} & =\min \sum_{t=k}^{k+H_{p}-1}\left[C_{\text {bess }}(t)+C_{\mathrm{DG}}(t)+C_{\mathrm{cur}}(t)\right], \\
C_{\mathrm{bess}}(t) & =\left[2 z_{\mathrm{bess}}(t)-P_{\text {bess }}(t)\right] \mathrm{OM}_{\mathrm{bess}} \Delta t, \\
C_{\mathrm{DG}}(t) & =C_{\mathrm{DG}}^{\mathrm{UD}}(t)+C_{\mathrm{DG}}^{\mathrm{OM}}(t)+C_{\mathrm{DG}}^{f}(t), \\
C_{\mathrm{DG}}^{\mathrm{UD}}(t) & =\sum_{n=1}^{N} \chi_{\mathrm{DG}, n}^{\mathrm{up}}(t)+\chi_{\mathrm{DG}, n}^{\mathrm{down}}(t), \\
C_{\mathrm{DG}}^{\mathrm{OM}}(t) & =\sum_{n=1}^{N} \mathrm{OM} \mathrm{DG}_{\mathrm{DG}, n}(t) \Delta t, \\
C_{\mathrm{DG}}^{f}(t) & =\sum_{n=1}^{N}\left(a \cdot P_{\mathrm{DG}, n}(t)+b\right) \Delta t, \\
C_{\mathrm{cur}}(t) & =\sum_{i=1}^{I} \alpha_{\mathrm{cur}, i}(t) \beta_{\mathrm{cur}, i}(t) P_{\mathrm{cur}, i}(t) \Delta t .
\end{aligned}
$$

The first objective function $C_{\text {total }}$, defined by equation (1), is formulated to minimize the total cost of the system's operation. According to equation (2), the cost of the ESS is mainly about the maintenance cost of the ESS due to frequent charge/discharge power. Equations (4)-(6) represent the calculation of the opening costs of the DGs $C_{\mathrm{DG}}^{\mathrm{UD}}(i)$, the maintenance cost of the $\mathrm{DGs} C_{\mathrm{DG}}^{\mathrm{OM}}(i)$, and the operating cost of the DGs $C_{\mathrm{DG}}^{f}(i)$, respectively. The penalty of load shedding, described by equation (2), represents the amount of load shedding $\beta_{\mathrm{cur}, i}(t) P_{\mathrm{cri}, i}(t)$ incurred by the unsatisfied power demand in the cutting load in each period.

It should be mentioned that $K$ represents the whole scheduling period and $k$ is defined as the current period of the scheduling procedure, while $t$ is the index for time step in a prediction horizon and $H_{p}$ is the length of a prediction horizon. The optimization problem is solved for each time step with the consideration of all constraints. It should be also noted that $\forall t \in\left[k, k+H_{p}-1\right], \forall k \in K$. The explanations of operation constraints are presented as follows:

$$
\begin{aligned}
& \delta_{\mathrm{DG}, n}(t) P_{\mathrm{DG}}^{\min } \leq P_{\mathrm{DG}, n}(t) \leq \delta_{\mathrm{DG}, n}(t) P_{\mathrm{DG}}^{\max }, \\
& -\Delta P_{\mathrm{DG}} \Delta t \leq P_{\mathrm{DG}, n}(t)-P_{\mathrm{DG}, n}(t-1) \leq \Delta P_{\mathrm{DG}} \Delta t, \\
& \delta_{\mathrm{DG}, n}(t)-\delta_{\mathrm{DG}, n}(t-1) \leq \delta_{\mathrm{DG}, n}\left(\tau_{1}\right), \\
& \tau_{1}=t+1, \ldots, \min \left(t+T^{\mathrm{up}}-1, k+H_{p}-1\right), \\
& \delta_{\mathrm{d} g, n}(t-1)-\delta_{\mathrm{d} g, n}(t) \leq 1-\delta_{\mathrm{DG}, n}\left(\tau_{2}\right), \\
& \tau_{2}=t+1, \ldots, \min \left(t+T^{\mathrm{down}}-1, k+H_{p}-1\right),
\end{aligned}
$$

$$
\begin{aligned}
& \chi_{\mathrm{DG}, n}^{\mathrm{up}}(t) \geq c_{\mathrm{DG}}^{\mathrm{up}}\left(\delta_{\mathrm{DG}, n}(t)-\delta_{\mathrm{DG}, n}(t-1)\right), \\
& \chi_{\mathrm{DG}, n}^{\mathrm{down}}(t) \geq c_{\mathrm{DG}}^{\mathrm{down}}\left(\delta_{\mathrm{DG}, n}(t-1)-\delta_{\mathrm{DG}, n}(t)\right), \\
& \chi_{\mathrm{DG}, n}^{\mathrm{up}}(t) \geq 0, \\
& \chi_{\mathrm{DG}, n}^{\text {down }}(t) \geq 0 .
\end{aligned}
$$

Constraints (8)-(14) are the constraints for the planner to determine the optimal scheme on the status of the DGs in each period. Constraints equations (8) and (9) denote the limits of the power ramp rate and the output of the DGs, respectively. Constraints (10) and (11) limit the shortest duration for which a DG must remain in either the on or off state. Constraints (12)-(14) are the detailed calculation method for the start-up/shut-down cost.

$$
\begin{aligned}
& P_{b}^{\min } \leq P_{\mathrm{ec}}(t)-P_{\mathrm{ed}}(t) \leq P_{b}^{\max }, \\
& E_{b}^{\min } \leq E_{b}(t) \leq E_{b}^{\max }, \\
& E_{b}\left(k+H_{p}-1\right)=E_{b}(0), \\
& E_{b}(t)=E_{b}(t-1)+\left(\frac{1}{\eta_{b}^{d}}\right)\left(P_{\mathrm{ec}}(t)-P_{\mathrm{ed}}(t)\right) \Delta t+ \\
& \left(\eta_{b}^{c}-\frac{1}{\eta_{b}^{d}}\right)\left(P_{\mathrm{ec}}(t)-P_{\mathrm{ed}}(t)\right) \delta_{b}(t) \Delta t-\varepsilon_{b} \Delta t .
\end{aligned}
$$

Constraints (15)-(20) correspond to the action of the planner on charging or discharging power of ESS. Constraints (15)-(16) impose the limit of charging/discharging power and the storage capacity, respectively. Equations (17) and (18) present the energy transition process of the ESS, where $\eta_{b}^{c}$ and $\eta_{b}^{d}$ are defined to distinguish the different charging and discharging efficiencies of the ESS. Because of the binary property of $\delta_{b}(t)$, the nonlinear equation (18) can be linearized as the equalities below using the big- $M$ method, in which an auxiliary variable $z_{b}(t)$ and extra constraints (19)-(25) are introduced to replace $\left(P_{\mathrm{ec}}(t)-P_{\mathrm{ed}}(t)\right) \delta_{b}(t)$.

$$
\begin{gathered}
-\delta_{b}(t) P_{b}^{\min } \leq P_{\mathrm{ec}}(t)-P_{\mathrm{ed}}(t)-P_{b}^{\mathrm{min}}, \\
P_{\mathrm{ec}}(t)-P_{\mathrm{ed}}(t)+\sigma \leq\left(P_{b}^{\mathrm{max}}+\sigma\right) \delta_{b}(t), \\
E_{b}(t)=E_{b}(t-1)+\left(\frac{1}{\eta_{b}^{d}}\right)\left(P_{\mathrm{ec}}(t)-P_{\mathrm{ed}}(t)\right) \Delta t \\
+\left(\eta_{b}^{c}-\frac{1}{\eta_{b}^{d}}\right) z_{b}(t) \Delta t-\varepsilon_{b} \Delta t, \\
z_{b}(t) \leq P_{b}^{\max } \delta_{b}(t), \\
z_{b}(t) \geq P_{b}^{\min } \delta_{b}(t),
\end{gathered}
$$




$$
\begin{gathered}
z_{b}(t) \leq P_{\mathrm{ec}}(t)-P_{\mathrm{ed}}(t)-P_{b}^{\mathrm{min}}\left(1-\delta_{b}(t)\right), \\
z_{b}(t) \geq P_{\mathrm{ec}}(t)-P_{\mathrm{ed}}(t)-P_{b}^{\max }\left(1-\delta_{b}(t)\right) .
\end{gathered}
$$

Equation (26) preserves the power input-output balance on each node, which ensures that each node remains as energy supply during each period. Constraint (27) presents the limit on the proportion of load shedding.

$$
\begin{aligned}
& P_{\mathrm{ec}}(t)+\sum_{i}^{I}\left(1-\beta_{\mathrm{cur}, i}(t)\right) P_{\mathrm{cur}, i}(t) \\
& =\sum_{n}^{N} P_{\mathrm{DG}, n}(t)+P_{\mathrm{ed}}(t)+P_{\mathrm{solar}}(t)+P_{\text {wind }}(t), \\
& \beta_{\mathrm{cur}}^{\min } \leq \beta_{\mathrm{cur}, i}(t) \leq \beta_{\mathrm{cur}}^{\max } .
\end{aligned}
$$

2.2. Presentation of the Uncertainty Set. In this section, we present the uncertainty sets of the RERs and the load demand, where the RERs include wind turbines and PV generators.

In RO theory, all possible uncertain events are classified into a deterministic set $\mathrm{W}$ in advance, and the optimal solution inhibits the possible adverse effects of each element in set W. To guarantee the robustness of the model, the uncertainty sets in this paper are proposed as follows:

$$
\begin{aligned}
W(t)= & \left\{\left\{w_{j}(t)\right\} \mid w_{j}^{e}(t)-w_{j}^{h}(t) \leq w_{j}(t) \leq\right. \\
& \left.w_{j}^{e}(t)+w_{j}^{h}(t), \forall t \in\left[k, k+H_{p}-1\right]\right\}, \quad j \in\{\text { solar, wind, cur }\},
\end{aligned}
$$

where $w_{j}^{e}(t)$ represents the expected value of the $j$-th uncertain parameter in period $t$ and $w_{j}^{h}(t)$ represents the deviation of the $j$-th uncertain parameter in period $t$. Generally, $w_{j}^{e}(t)$ can be gained through a statistical analysis of historical data, while $w_{j}^{h}(t)$ can be generated from online method for prediction.

It is worth mentioning that all elements in $\mathrm{W}$ are independent random variables with the same distribution form but without the information of the distribution. According to the Central Limit Theorem, the uncertain set is transformed into the below formulation based on the theory in [18].

$$
W(t)=\left\{w_{j}(t) \mid \sum_{j} \frac{\left|w_{j}(t)-w_{j}^{e}(t)\right|}{w_{j}^{h}(t)} \leq \Gamma\right\},
$$

where $\Gamma$ is defined as the price of robustness with the limitation in $[0, J] . J$ represents the number of uncertain variables. It can be specific to conclude that the deviation between the real data and the predicted data will disappear when $\Gamma=0$, meaning that the proposed problem changes into a deterministic problem. On the contrary, the system is deemed to be in the worst situation when $\Gamma=J$ resulted in a conservative solution generating from the proposed model.

\section{The Framework of RMPC Based on Interval Prediction}

3.1. Structure of Traditional MPC for Energy Management. Model Predictive Control is a novel control method that converts an infinite long open-loop optimization into a limited long closed-loop optimization at each sample time, using the retreated horizon strategy and considering the dynamic performance of the system, the control objectives, and the constraints [19]. By solving a closed-loop optimal control problem, MPC can reduce the deviation between the future output and reference trajectory [20]. In terms of the energy management of IMGs, this means that, at the current point in time, optimal scheduling for 24 hours is generated based on the predictions of the upcoming demand and production from renewable energy units. Some researchers have applied MPC in optimal energy management of power systems and have obtained promising results [13-17].

The schematic overview of the traditional MPC is divided into four aspects as illustrated in Figure 1. The first aspect of the traditional MPC is the data forecasting of the power system including the output of RERs and the demand of load for a period of time. Generally, the OL method is used to predict the future information of multiple uncertainty sources. In the second aspect, the model of the EMS and IMGs system constraints is formulated based on the system state measurement. The third aspect is the process of optimization to minimize the total operating costs over a given time horizon in the first aspect. It should be mentioned that implementation of the proposed optimization approach is always based on the latest information and only the first interval scheduling is implemented. With the prescheduling generated from the third aspect, the feedback correction strategy is applied to adjust the output of the units to balance the difference between the forecast values and the actual values in the fourth aspect. The four aspects interact with each other and are implemented sequentially. The process of the closed-loop is repeated with the time step forward so that control remains optimal and tolerates the uncertainty in RES and load.

However, the prediction of the uncertainties in the traditional MPC is usually point forecasting resulting in a great deviation from the real data with the time horizon 


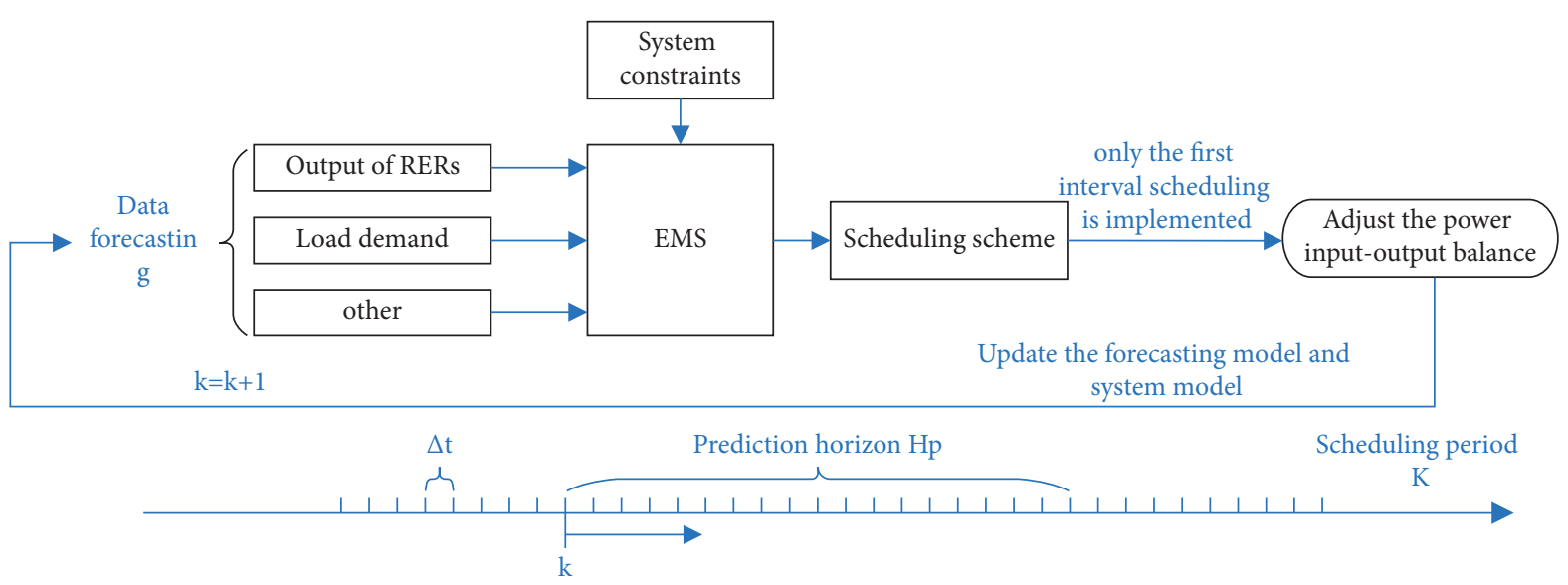

FIGURE 1: The traditional schematic overview of the traditional MPC.

moving forward. To ensure the stability and the reliability of electric power supply under MPC strategy, the interval prediction is proposed to obtain the boundaries of state variables instead of point forecasting. Furthermore, the optimization model is reformulated into a robust model based on interval prediction to alleviate the interference of the power output and the load demand. More details about the RMPC strategy are illustrated in the following sections.

3.2. The RMPC Strategy Based on Interval Prediction. Recently, a few studies have investigated RERs output and load demand forecasting through interval prediction $[21,22]$. Interval prediction is a prediction of an interval in which a real value in the future will drop, with a certain probability called the confidence level. In interval prediction, interval arithmetic deals with the uncertain inputs that lie within a certain interval and enables the direct computation towards the bounds of state variables. With the upper and lower bounds of uncertain parameters in power systems, more information about the RERs and load demand can be obtained for EMS to DA scheduling.

In interval prediction, the relationship between the input variables and the output variables is presented as follows:

$$
\left\{\begin{array}{l}
t_{j}=y_{j}+\varepsilon_{j}, \\
y_{j}=f\left(x_{j}\right), \quad j=1,2, \ldots, J
\end{array}\right.
$$

where $t_{j}$ is the uncertainty variable and $x_{j}$ is the input variable. $y_{j}$ is a theoretically perfect and unbiased regression. $\varepsilon_{j}$ is the error between $t_{j}$ and $y_{j}$. In application, the perfect regression is estimated from the corresponding regression model $\hat{y}_{i}$ as follows:

$$
t_{i}-\hat{y}_{i}=\left[y_{i}-\hat{y}_{i}\right]+\varepsilon_{i} .
$$

Consequently, interval prediction is the estimation for the error between the true value and the forecasting value with Confidence Interval (CI) and Prediction Interval (PI) in quantifying the uncertainty of the error between $\widehat{y}_{i}$ and $y_{i}$ and the error between $t_{i}$ and $\hat{y}_{i}$, respectively.

Generally, the error $\varepsilon_{i}$ and $\left[y_{i}-\widehat{y}_{i}\right]$ are independent so that the variance of the true value and the forecasting value is formulated as follows:

$$
\sigma_{i}^{2}=\sigma_{\hat{y}_{i}}^{2}+\sigma_{\widehat{\varepsilon}_{i}}^{2}
$$

where $\sigma_{\widehat{\varepsilon}}^{2}$ is the measurement of the variance of error and $\sigma_{\widehat{v}}^{2}$ is the measurement of the variance of error. Because the errors of prediction follow a normal distribution with a mean of zero, the true value is fluctuant within the interval of the forecasting value. Hence, the PI is formulated as follows:

$$
\mathrm{PI}=\widehat{y}_{i} \pm z^{1-(\alpha / 2)} \sqrt{\sigma_{\widehat{y}_{i}}^{2}+\sigma_{\widehat{\varepsilon}_{i}}^{2}}
$$

where $(1-\alpha) \times 100 \%$ is the confidence level, which means that the forecasting value has a $(1-\alpha) \%$ chance fall in the PI; $\widehat{y}_{i}$ is the regression value of point forecasting.

In this paper, the OL method is used to predict the future information of multiple uncertainty sources in the RMPC based on interval prediction, including the output of RERs and load demand. The Deep Belief Network (DBN) is introduced as a basis of the OL method. Since the implementation of the DBN method has been extensively reported in the literature, a detailed solution process is omitted in this manuscript for the sake of contribution clarification. For more details, the interested reader is referred to $[23,24]$.

Based on the theory of interval prediction, the robust optimization ( $\mathrm{RO}$ ) method is proposed to address the fluctuation of uncertainties in traditional MPC. According to the thesis in [18] and the uncertainty set presented in (29), the constraint (26) is reformulated into the inequation (34) and several constraints are added in the model as follows: 


$$
\begin{aligned}
& \left\{\begin{array}{l}
\sum_{i}^{I} P_{\text {cur }, i}^{e}(t)\left(1-\beta_{\text {cur }, i}(t)\right)+z_{\text {balance } 1}(t) \Gamma_{\text {balance } 1}(t)+\sum_{i}^{I} p_{\text {balance } 1, i}(t)+z_{\text {balance } 2}(t) \Gamma_{\text {balance } 2}(t) \\
+\sum_{j=1}^{3} p_{\text {balance } 2, j}(t) \leq P_{\text {solar }}^{e}(t)+P_{\text {wind }}^{e}(t)-\sum_{i}^{I} P_{\text {cur }, i}^{e}(t)+P_{\text {ed }}(t)-P_{\mathrm{ec}}(t)+\sum_{n}^{N} P_{\mathrm{DG}, n}(t),
\end{array}\right. \\
& -y_{\text {balance } 1, i}(t) \leq 1-\beta_{\text {cur }, i}(t) \leq y_{\text {balance } 1, i}(t), \quad i \in I, \\
& z_{\text {balance } 1}(t)+p_{\text {balance } 1, i}(t) \geq P_{\text {cur }, i}^{h}(t) y_{\text {balance } 1, i}(t), \quad i \in I, \\
& z_{\text {balance } 2}(t)+p_{\text {balance } 2,1}(t) \geq P_{\text {solar }}^{h}(t) \\
& z_{\text {balance } 2}(t)+p_{\text {balance } 2,2}(t) \geq P_{\text {wind }}^{h}(t), \\
& z_{\text {balance } 2}(t)+p_{\text {balance } 2,3}(t) \geq P_{\text {cur }, i}^{h}(t), \quad i \in I, \\
& P_{\text {balance } 1, i}(t) \geq 0, \quad i \in I \text {, } \\
& P_{\text {balance } 2, j}(t) \geq 0, \quad j=1,2,3 \text {, } \\
& z_{\text {balance } 1}(t) \geq 0 \text {, } \\
& z_{\text {balance } 2}(t) \geq 0 \text {, }
\end{aligned}
$$

where $z_{\text {balance } 1}(t), z_{\text {balance } 2}(t), p_{\text {balance } 1, i}(t), p_{\text {balance } 2, j}(t)$, and $y_{\text {balance } 1, i}(t)$ are additional variables. $\Gamma_{\text {balance } 1}(k)$ and $\Gamma_{\text {balance } 2}(t)$ indicate the degree of influence from uncertainty and are within $[0, I]$ and $[0,3]$, respectively. Constraints (35)-(42) are additional constraints to illustrate the relationship between the auxiliary variables $z_{\text {balance } 1}(t)$, $z_{\text {balance } 2}(t), p_{\text {balance } 1, i}(t), p_{\text {balance } 2, j}(t)$, and $y_{\text {balance } 1, i}(t)$ and the original variables $P_{\text {cur }, i}(t), P_{\text {solar }}(t)$, and $P_{\text {wind }}(t)$.

Due to the uncertainty variables in the objective function (1), the objective function is reformulated into the inequation as follows:

$$
\begin{aligned}
& \sum_{i}^{I} \alpha_{\mathrm{cur}, i}(t) \beta_{\mathrm{cur}, i}(t) P_{\mathrm{cur}, i}^{h}(t) \Delta t+C_{\mathrm{bess}}(t)+C_{\mathrm{DG}}(t) \\
& -z_{\mathrm{goal}}(t)+z_{\mathrm{cur}}(t) \Gamma_{\mathrm{cur}}(t)+\sum_{i}^{I} p_{\mathrm{cur}, i}^{h}(t) \leq 0 \\
& z_{\mathrm{cur}}(t)+p_{\mathrm{cur}, i}(t) \geq \alpha_{\mathrm{cur}, i}(t) P_{\mathrm{cur}, i}^{e}(t) y_{\mathrm{cur}, i}(t) \Delta t, \quad i \in I \\
& -y_{\mathrm{cur}, i}(t) \leq \beta_{\mathrm{cur}, i}(t) \leq y_{\mathrm{cur}, i}(t), \quad i \in I \\
& p_{\mathrm{cur}, i}(t) \geq 0, \quad i \in I \\
& z_{\mathrm{cur}}(t) \geq 0,
\end{aligned}
$$

where $z_{\text {cur }}(t), p_{\text {cur }, i}(t)$, and $y_{\text {cur }, i}(t)$ are additional variables. $\Gamma_{\text {cur }}(t)$ quantifies the effect of uncertainty satisfying $\Gamma_{\text {cur }}(t) \in[0, I]$. Constraints $(44)-(47)$ are additional constraints to illustrate the relationship between the auxiliary variables $z_{\text {cur }}(t), p_{\text {cur }, i}(t)$, and $y_{\text {cur }, i}(t)$ and the original variables $P_{\text {cur }, i}(t)$ and $\beta_{\text {cur }, i}(t)$.

To sum up, the energy management of IMGs problem is reformulated into the robust model as below based on the method presented in [18]. To simplify the solution procedure, $\Gamma$ is built to present the degree of robustness of the model by normalizing. When $\Gamma=0$, the problem is degenerate into the deterministic model. On the other hand, $\Gamma=0$ indicates that the solution generated from the model will be the most conservative. Otherwise, $\Gamma$ is within $[0,1]$.

$$
\begin{aligned}
& \min z_{\text {goal }}(k) \\
& \text { s.t. }(8)-(25),(4)-(47),(27) .
\end{aligned}
$$

3.3. Solution Process. With the formulated model in Sections 2 and 3.2, the RMPC controller calculates an optimal control measure sequentially for each step in the scheduling horizon. Once new measurements of the current step and predictions of the scheduling horizon are attained, a new optimization problem with updated states is solved. In a prediction horizon, only the first interval scheduling is implemented. With such an optimization approach, the total operational cost over the prediction horizon is minimized.

To clarify the logic of the whole method, a flowchart about the implementation framework of the RMPC method is shown below in this section. It is noted that the optimization problem can be solved by commercial mixed-integer programming solvers such as CPLEX and Gurobi (Algorithm 1). 
while $k<K$ do

(1) Measure the current system state: the switching status of DGs $\delta_{\mathrm{DG}, n}(k)$ and the capacity of storage $E_{b}(k)$;

(2) Read the updated historical data, apply OL method, and forecast future interval based on the new updated values;

(3) Optimize RO model from section for time step $k$ for entire prediction horizon $H_{p}$ to obtain DA scheduling;

(4) Implement the first interval scheduling plan;

(5) Receiving real-time data of RERs and load demand, optimize energy management model in the second section for real-time (RT) scheduling;

(6) Implement new control actions; end while

return a series of DA scheduling $\delta_{\mathrm{DG}, n}, E_{b}(k)$, and RT scheduling $P_{\mathrm{DG}, n}, P_{\mathrm{ec}}, P_{\mathrm{ed}}, \delta_{b}$ and $\beta_{\mathrm{cur}, i}$.

Algorithm 1: Framework of the RMPC method.

\section{Case Studies}

4.1. Experiment Settings. All simulation tests are coded in IBM ILOG CPLEX V12.80 and run on a 64-bit Windows $\mathrm{PC}$ with four Intel Core i7-8550U CPU at $1.80 \mathrm{GHz}$ and 16.0 GB of RAM. The whole day is divided into 24 successive periods by the hour. The case studies are carried out on the IMG system with 4 DGs, 1 storage, and several routers as shown in Figure 2. The four DGs have the same parameters and property. The data on the output of RERs and the load demand of the critical load and cutting load are retrieved from the Global Energy Forecasting Competition 2014 and as shown in Figure 3, where each predicted uncertainty interval is set to $0 \%, 2.5 \%$, and $5.0 \%$ of the nominal value. The cutting rate of load shedding $\beta_{\text {cur, } i}$ is limited in $[0,0.3]$. The other experimental parameters are listed in Tables 1 and 2.

According to these case studies, the proposed model is compared with the traditional MPC approach and the Hard Charging (HC) method to evaluate its superiority. Besides, a sensitivity analysis about the parameters of the RMPC method is conducted to explore the most appropriate setting for IMGs. In the following, the length of a prediction horizon $H_{p}$ is set as 10 unless otherwise stated.

4.2. Performance of the RO Model. To investigate the performance of the RO model based on interval prediction in the MPC method, in this section, we compared the output of DGs, the states of the ESS, and the operation cost under the scheduling horizon in the RMPC method and the traditional MPC method. In terms of energy management, $\Delta t$ is set for 1 hour. The comparison results are reported in Figures 4-7.

The "Width of Interval (WI)" in Figure 4 presents the error range of prediction. For instance, the $\mathrm{WI} 2=2.50 \%$ means the forecasting value $x$ is within $[x-0.025 x, x+0.025 x]$. It should be mentioned that if $\mathrm{WI}=0$, the uncertain problem is transformed into a deterministic problem. Meanwhile, $\Gamma=0$ indicates that the solution generated from the RMPC method is equal to the traditional MPC method. The change tendency of operation cost with $\Gamma$ increases is described in Figure 4 . We can observe that the change of cost calculated from the RMPC method firstly decreases and then decreases as $\Gamma$ becomes bigger. Compared with the traditional MPC method, when

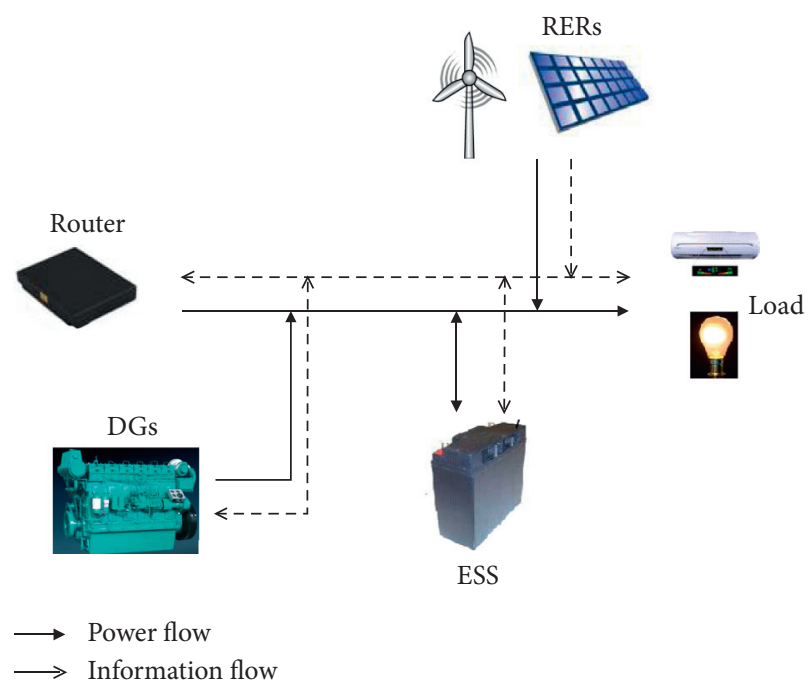

FIGURE 2: Islanded microgrid test system.

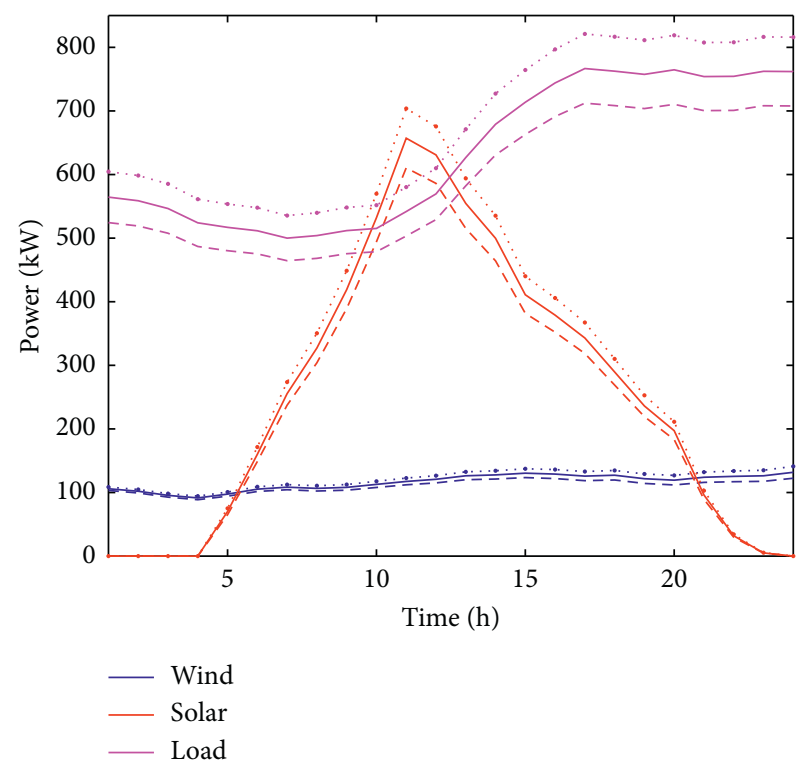

FIGURE 3: Fluctuation ranges of uncertain parameters with uncertainty interval set to $2.5 \%$. 
TABle 1: Operating parameters of the DGs.

\begin{tabular}{|c|c|c|c|c|c|c|c|c|c|}
\hline$P_{\mathrm{DG}}^{\max } /(\mathrm{kW})$ & $P_{\mathrm{DG}}^{\min }(\mathrm{kW})$ & $\Delta P_{\mathrm{DG}}(\mathrm{kW})$ & $T^{\mathrm{up}}(\mathrm{h})$ & $T^{\text {down }}(\mathrm{h})$ & $\mathrm{a}$ & $\mathrm{b}$ & $c_{\mathrm{d} g}^{\mathrm{up}}$ & $c_{\mathrm{d} g}^{\text {down }}$ & $\mathrm{OM}_{\mathrm{d} g}$ \\
\hline 180 & 150 & 150 & 2 & 2 & 0.000469 & 0.496 & 3.10 & 3.36 & 0.9 \\
\hline
\end{tabular}

TABle 2: Parameter settings of the ESS.

\begin{tabular}{lccccccc}
\hline$E_{b}^{\max } /(\mathrm{kW})$ & $E_{b}^{\min }(\mathrm{kW})$ & $P_{b}^{\max }(\mathrm{kW})$ & $P_{b}^{\min }(\mathrm{kW})$ & et $a_{b}^{c}$ & et $a_{b}^{d}$ & $\varepsilon_{b}$ & $\mathrm{OM}_{b}$ \\
\hline 800 & 40 & 400 & -400 & $95 \%$ & $95 \%$ & 0.02 & 0.05 \\
\hline
\end{tabular}

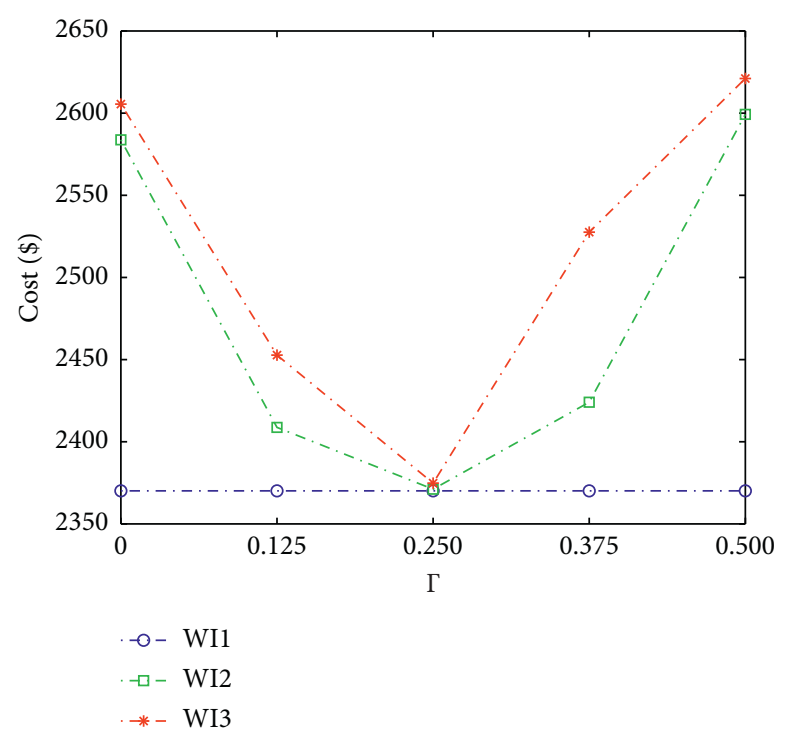

FIGURE 4: Comparison of the operation cost between RMPC method and traditional MPC.

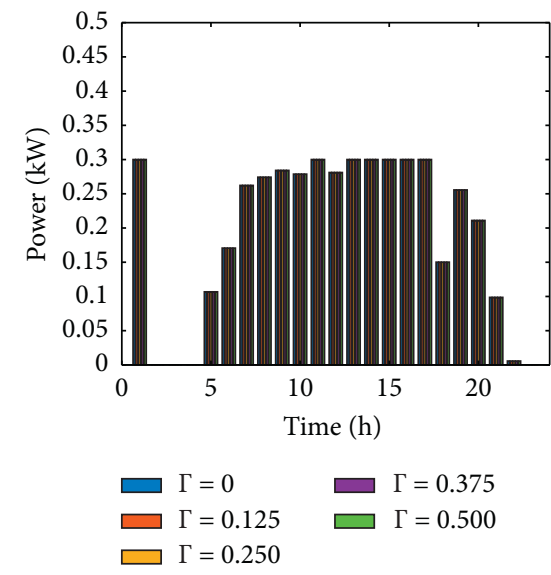

(a)

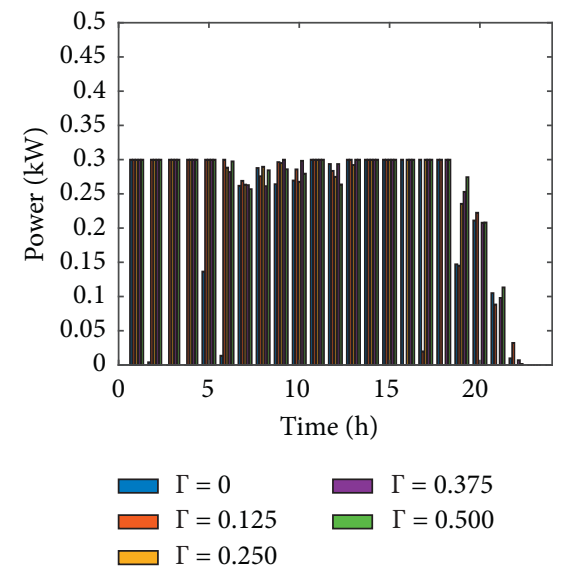

(b)

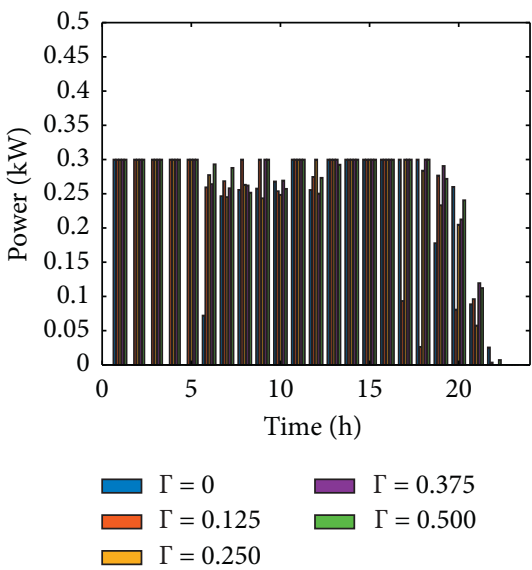

(c)

FIGURE 5: Comparison of the load shedding between the RMPC method and the traditional MPC. (a) WI1 $=0.0$. (b) WI2 $=2.5 \%$. (c) $\mathrm{WI} 3=5.0 \%$.

$\Gamma \leq 0.250$, the expense is cheaper; otherwise, the payment becomes even greater when $\Gamma>0.500$. With the operation of load shedding, DGs and ESS presented in Figures 5-7; it can be explained by the fact that as $\Gamma$ increases, the decision- makers become more conservative so that more measures are implemented to deal with a shortage of energy with the consideration of extreme instability. Besides, the operation cost is increased as the WI becomes greater. The 


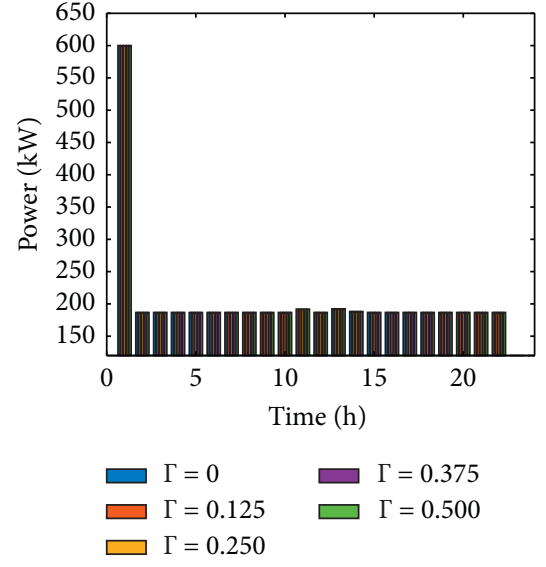

(a)

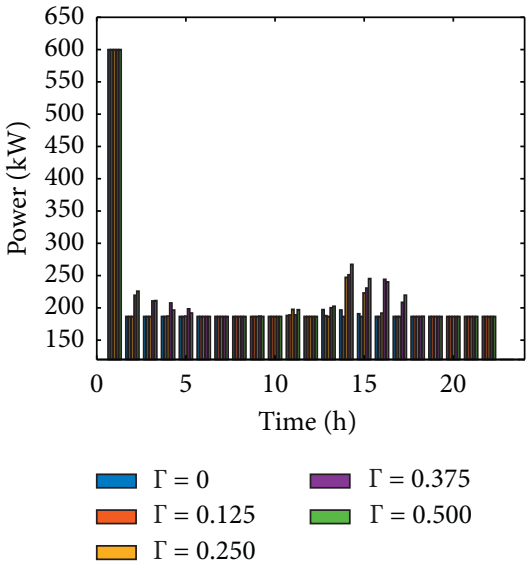

(b)

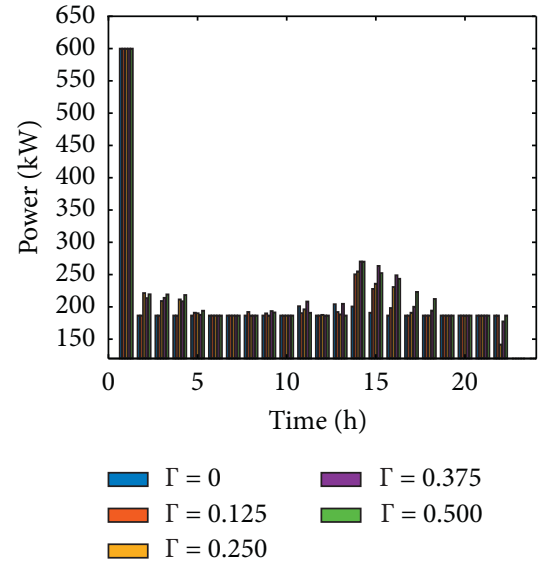

(c)

FIgURE 6: Comparison of the output of DGs between the RMPC method and the traditional MPC. (a) WI1 $=0.0$. (b) WI2 $=2.5 \%$. (c) $\mathrm{WI} 3=5.0 \%$.

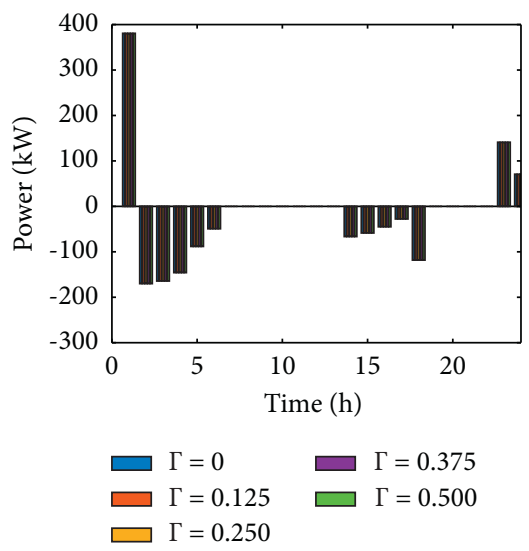

(a)

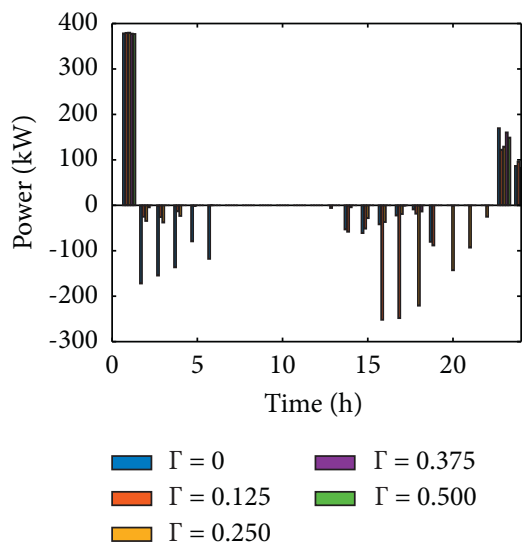

(b)

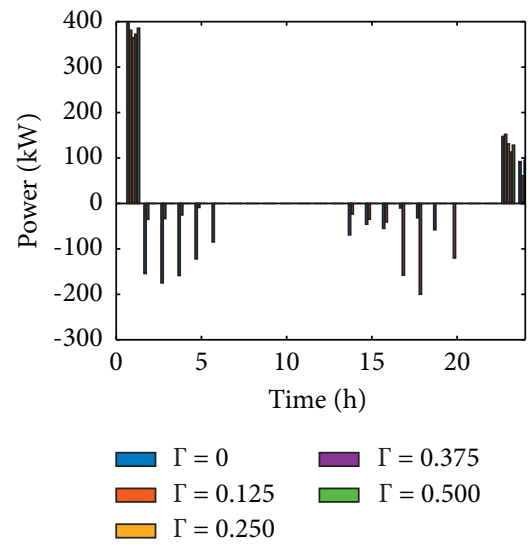

(c)

FIgURE 7: Comparison of the discharging of ESS between the RMPC method and the traditional MPC. (a) WI1 $=0.0 \%$. (b) WI2 $=2.5 \%$. (c) $\mathrm{WI} 3=5.0 \%$.

reason is that the decision-makers should take several measures to compensate for the interference from the growth error of prediction such as extended boot time of DGs, raising DGs output, and continuous discharge from ESS. Nevertheless, such a method can reduce about $10.37 \%$ of the cost. Such observation confirms the challenge and the importance of selecting proper $\Gamma$ of RMPC to effectively mitigate the impact from uncertainty with less operation cost.

4.3. Performance of the RMPC Method Based on Interval Prediction. In this section, we evaluate the performance of the RMPC method on reducing the operation cost of energy management comparing with the HC method. HC method is a mature approach for energy management and DA scheduling applying in the industrial circle. The solution process is presented in Figure 8 . It will be instructive to review the objective function of the model and the operation of DA scheduling, that is, the operation cost of DGs obtained from the two methods before analyzing the advantages. The results are shown in Figures 9 and 10.

To obtain a robust scheduling plan, the gama in RMPC is set as 0.5 and the WI is set as $5 \%$ to generate the most conservative solution. As presented in Figure 9, the operation cost most of the time in the RMPC method is less than the HC method. The whole cost in the scheduling horizon of RMPC is 2621.08 \$, which is reduced to $16.61 \%$ compare with the HC method. To illustrate the drastic differences between these two approaches, we presented the histogram of the output of DGs in Figure 11 and the line chart of charging/discharging of ESS in Figure 10. It can be seen that the power generated from DGs and discharging power in the RMPC method is less than the HC method in the mass except at a certain time. However, the operation in RMPC is still sometimes spending more than the $\mathrm{HC}$ method in the 


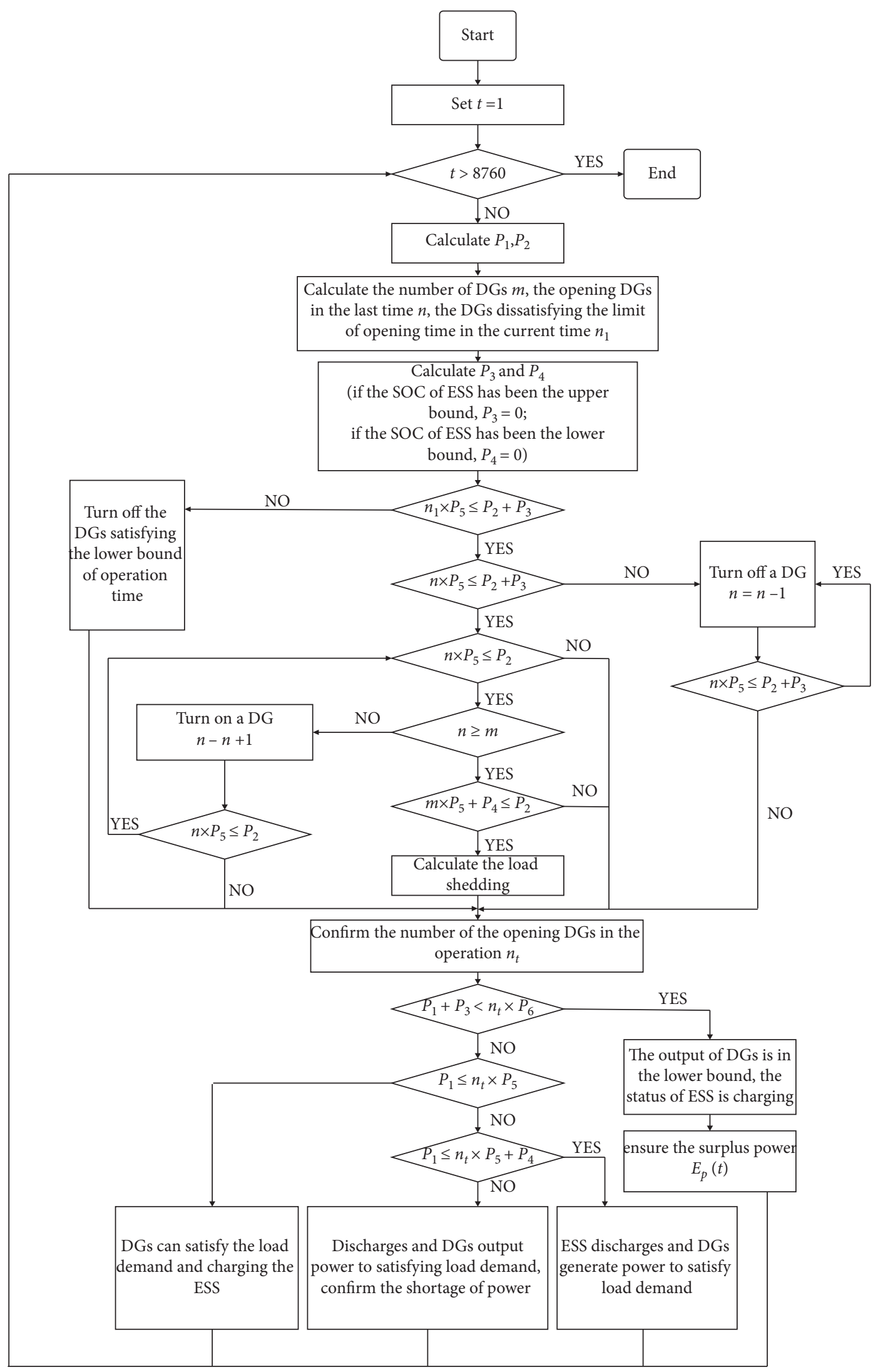

FIGURE 8: The solution procedure of the HC method. 


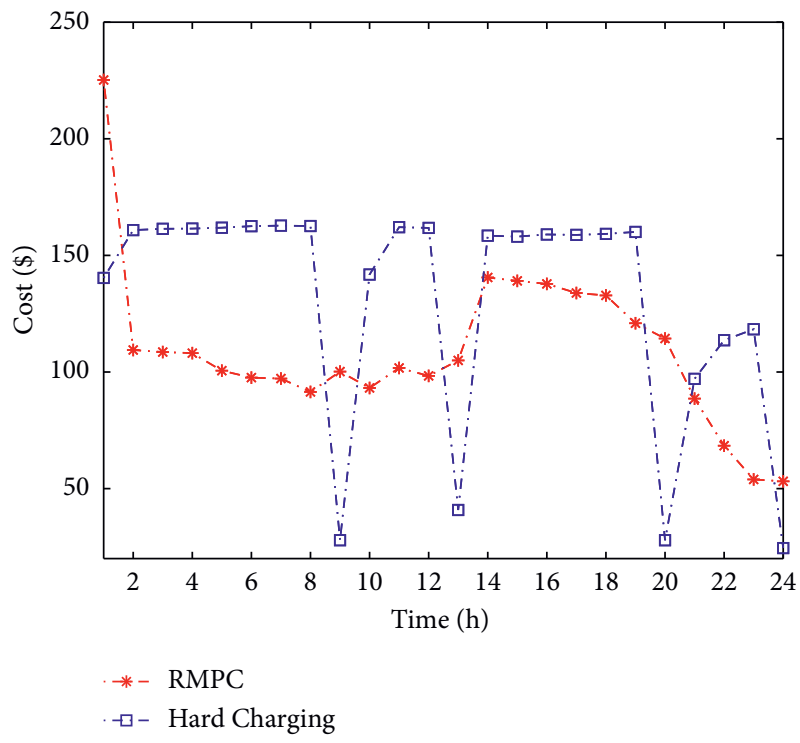

FIGURE 9: Comparison of operation cost between RMPC and HC method.

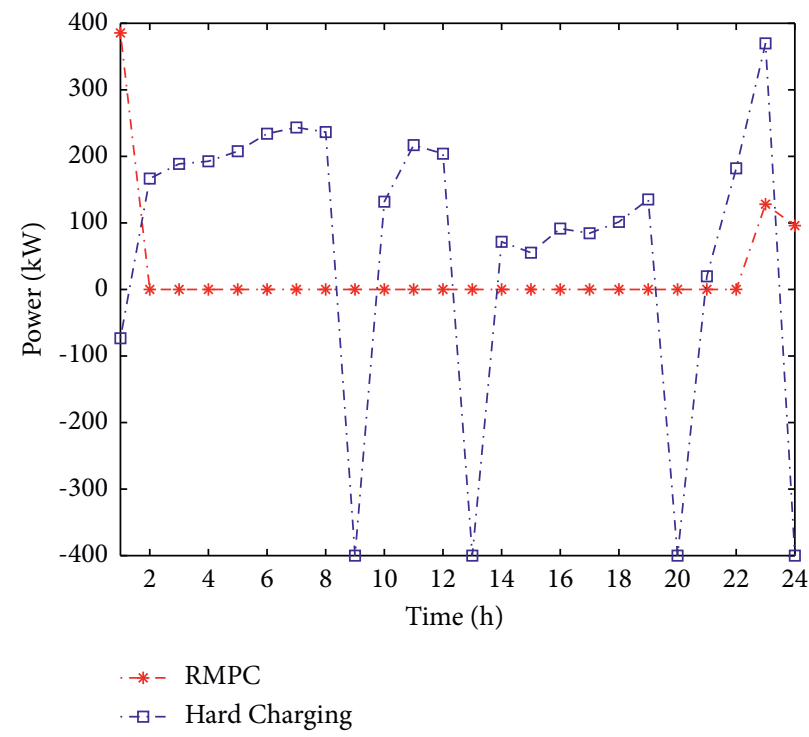

FIGURE 10: Comparison of the discharging of ESS between RMPC and HC method.

whole scheduling horizon such as at 9:00, 13:00, and 20:00 in Figure 10. This is because the RMPC is more conservative than the HC method every time so that even the prediction is precise, the output of DGs and the discharging power are still overflowing to avoid an energy supply shortage. The reason why the RMPC method is economical is that the setting of operation in the HC method is based on the experience of a decision-maker without an optimal solution from the mathematical model. Furthermore, the information in interval prediction would not be of good use in the HC method as the processing of the HC method presented in Figure 8. Given the fact that the RMPC method is more flexible than the $\mathrm{HC}$ method, we can conclude that by modeling and implementing the RMPC method based on interval prediction as an energy management approach, we can derive a cost-effective DA and RT scheduling that significantly outperforms those obtained from the traditional method. 


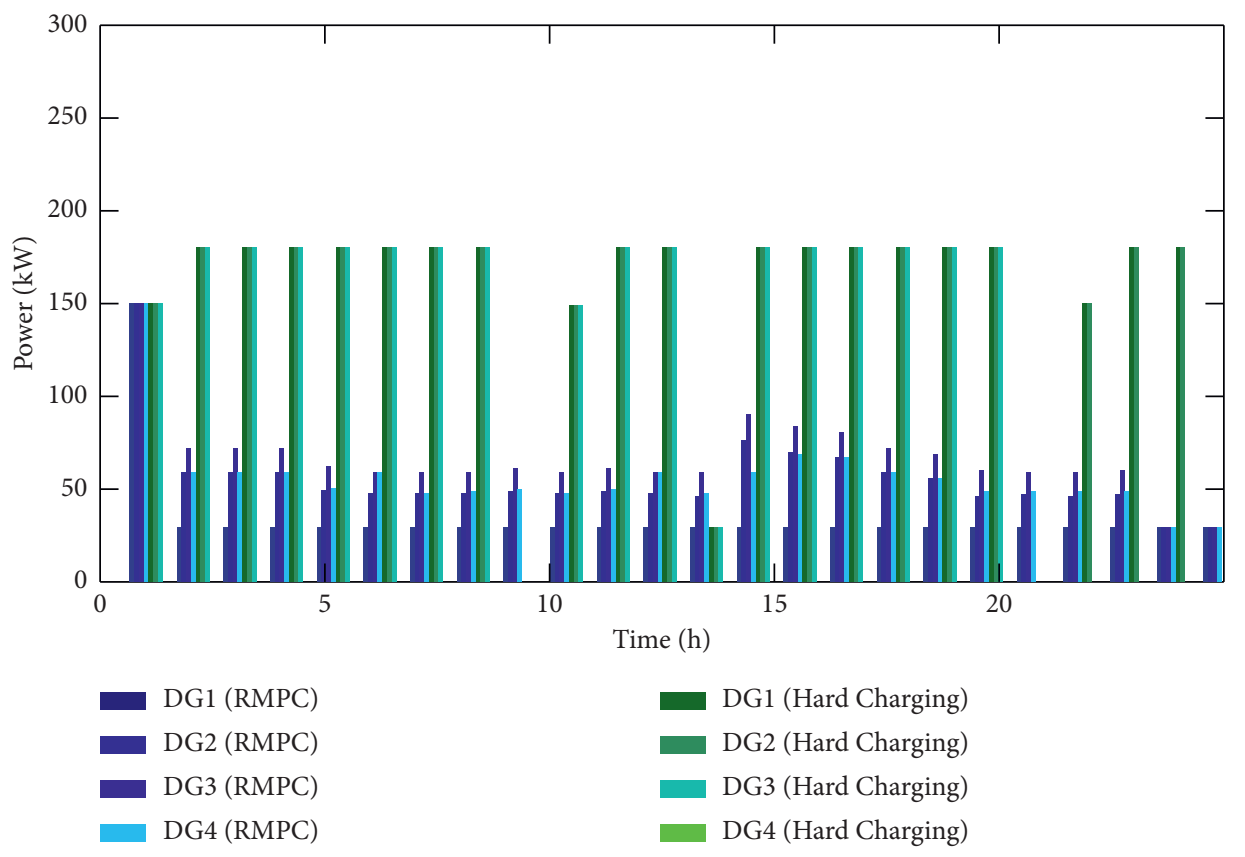

FIGURE 11: Comparison of the output of DGs between RMPC and HC method.

\section{Conclusions and Future Work}

This paper investigates the energy management problem in DA scheduling and RT scheduling for IMG. An RMPC method based on interval prediction is proposed to address uncertainties in information such as the generation of RERs and load demand to increase flexibility. The price of robustness is formulated to quantify the fluctuation of uncertainties. The OL method is utilized to predict future information for interval prediction of the system in the decision-making process. The length of the prediction horizon is selected according to the calculation burden, operational cost, and wind power curtailment. The proposed model is applied to the modified test system. The results demonstrate the effectiveness of the proposed model and method, showing the improvement of the control accuracy for the electricity supply network under uncertain conditions. In particular, a performance comparison among the RMPC, the traditional MPC, and the HC method is implemented, and sensitivity analysis of $\Gamma$ is performed as well. In the future, associated failures in DGs or transmission lines will be investigated when designing reliable energy management planning. Besides, application-related research of RO, such as distributionally robust optimization method, will be conducted in the field of MPC method for energy management. And the solution algorithm will be further improved to promote computational efficiency in prediction.

\section{Nomenclature}

Indices and sets

$K, k$ : The total number of time steps in the scheduling horizon the current time step of the scheduling horizon
$N, n$ : Set of Distributed Generators and their indexes

$I, i$ : Set of cutting loads and load index.

Parameters

$\Delta t: \quad$ Length of each period

$\Delta P_{\mathrm{DG}}: \quad$ Maximum power ramp rate of Distributed Generators (DGs)

$P_{\mathrm{DG}}^{\min } / P_{\mathrm{DG}}^{\max }$ : Minimum and maximum power of DGs

$c_{\mathrm{DG}}^{\mathrm{up}} / c_{\mathrm{DG}}^{\mathrm{down}}:$ Switching cost coefficients of DGs

$T^{\mathrm{up}} / T^{\text {down }}:$ Minimum up and downtime of DGs

$\mathrm{OM}_{\mathrm{DG}}$ : $\quad$ Maintenance cost of DGs

$a, b: \quad$ Fuel cost coefficients of DGs

$P_{b}^{\min } / P_{b}^{\max }:$ Minimum and maximum charging/discharging power levels of the Energy Storage System (ESS)

$E_{b}^{\min } / E_{b}^{\max }$ : Minimum and maximum capacity limit of the ESS

$\beta_{\text {cur }}^{\min } / \beta_{\text {cur }}^{\max }:$ Minimum and maximum cutting rate of the shedding load $i$

$\alpha_{\text {cur }, i}: \quad$ The penalty price of load shedding

$\mathrm{OM}_{b}$ : $\quad$ Maintenance cost of the ESS

$\eta_{b}^{c} / \eta_{b}^{d}$ : Charging and discharging efficiency coefficients of the ESS

$\varepsilon_{b}: \quad$ Self-discharge power of the ESS

$H_{p}: \quad$ The length of time window.

Uncertainty sets

$P_{\text {solar: }}$ : Actual solar power levels

$P_{\text {solar }}^{e}$ : Expected value of the forecast solar power levels

$P$ solar : Deviations from the expected value of the forecast solar power levels

$P_{\text {solar }}$ : Actual solar power levels

$P_{\text {solar }}^{e}$ : Expected value of the forecast solar power levels

$P$ solar : Deviations from the expected value of the forecast solar power levels

$P_{\text {wind }}$ : Actual wind power levels 
$P_{\text {wind }}^{e}$ : Expected value of the forecast wind power levels

$P_{\text {wind }}^{h}$ : Deviations from the expected value of the forecast wind power levels

$P_{\text {cur }, i}:$ Actual load demand of the cutting load $i$

$P_{\text {cur }, i}^{e}$ : Expected values of the forecast load demand of the cutting load $i$

$P_{\text {cur }, i}^{h}$ : Deviations from the expected value of the forecast load demand of the cutting load $i$.

Variables

$\delta_{\mathrm{DG}, n}:$ On/off status of DGs $n$

$P_{\mathrm{DG}, n}$ : Output power of DGs $n$

$\chi_{\mathrm{DG}, n}^{\mathrm{up}}$ : Switching on costs of DGs $n$

$\chi_{\mathrm{DG}, n}^{\mathrm{down}}$ : Switching off costs of DGs $n$

$P_{\mathrm{ec}}$ : Charging power levels of the ESS

$P_{\mathrm{ed}}$ : Discharging power levels of the ESS

$\delta_{b}$ : $\quad$ Status of the ESS

$E_{b}$ : Capacity of the ESS

$z_{b}$ : Extra instrumental variable for the ESS

$\beta_{\text {cur }, i}$ : Cutting rate of the shedding load $i$.

\section{Data Availability}

The data used to support the findings of this study have been deposited in the Global Energy Forecasting Competition 2014.

\section{Conflicts of Interest}

The authors declare that they have no conflicts of interest.

\section{Acknowledgments}

This work was supported by the National Natural Science Foundation of China (Grants nos. 71901210 and 61973310).

\section{References}

[1] F. Katiraei, R. Iravani, N. Hatziargyriou, and A. Dimeas, "Microgrids management," IEEE Power and Energy Magazine, vol. 6, no. 3, pp. 54-65, 2008.

[2] N. Mazzi and P. Pinson, "Wind power in electricity markets and the value of forecasting," Renewable Energy Forecasting, vol. 2017, pp. 259-278, 2017.

[3] B. F. Hobbs, "Optimization methods for electric utility resource planning," European Journal of Operational Research, vol. 83, no. 1, pp. 1-20, 1995.

[4] Y. Wang, Y. Ma, F. Song et al., "Economic and efficient multiobjective operation optimization of integrated energy system considering electro-thermal demand response," Energy, vol. 205, Article ID 118022, 2020.

[5] L. Ji, X. Liang, Y. Xie, G. Huang, and B. Wang, "Optimal design and sensitivity analysis of the stand-alone hybrid energy system with pv and biomass-chp for remote villages," Energy, vol. 225, Article ID 120323, 2021.

[6] A. Lorestani, G. B. Gharehpetian, and M. H. Nazari, "Optimal sizing and techno-economic analysis of energy- and costefficient standalone multi-carrier microgrid," Energy, vol. 178, pp. 751-764, 2019.

[7] S. Peláez-Peláez, A. Colmenar-Santos, C. Pérez-Molina, A.-E. Rosales, and E. Rosales-Asensio, "Techno-economic analysis of a heat and power combination system based on hybrid photovoltaic-fuel cell systems using hydrogen as an energy vector," Energy, vol. 224, Article ID 120110, 2021.

[8] A. Maulik and D. Das, "Optimal operation of droop-controlled islanded microgrids," IEEE Trans. Sustainable Energy, vol. 9, no. 3, pp. 1337-1348, 2017.

[9] Z. Wang, B. Chen, J. Wang, J. Kim, and M. M. Begovic, "Robust optimization based optimal dg placement in microgrids," IEEE Transactions on Smart Grid, vol. 5, no. 5, pp. 2173-2182, 2014.

[10] X. Liu and X. Kong, "Nonlinear model predictive control for dfig-based wind power generation," IEEE Transactions on Automation Science and Engineering, vol. 11, no. 4, pp. 1046-1055, 2013.

[11] X. Liu, D. Jiang, and K. Y. Lee, "Decentralized fuzzy mpc on spatial power control of a large phwr," IEEE Transactions on Nuclear Science, vol. 63, no. 4, pp. 2343-2351, 2016.

[12] A. Parisio, E. Rikos, and L. Glielmo, "A model predictive control approach to microgrid operation optimization," IEEE Transactions on Control Systems Technology, vol. 22, no. 5, pp. 1813-1827, 2014.

[13] A. Turk, Q. Wu, and M. Zhang, "Model predictive control based real-time scheduling for balancing multiple uncertainties in integrated energy system with power-to-x," International Journal of Electrical Power \& Energy Systems, vol. 130, Article ID 107015, 2021.

[14] L. Ye, C. Zhang, Y. Tang et al., "Hierarchical model predictive control strategy based on dynamic active power dispatch for wind power cluster integration," IEEE Transactions on Power Systems, vol. 34, no. 6, pp. 4617-4629, 2019.

[15] L. Yin and Z. Su, "Multi-step depth model predictive control for photovoltaic power systems based on maximum power point tracking techniques," International Journal of Electrical Power \& Energy Systems, vol. 131, Article ID 107075, 2021.

[16] J. Hu, Y. Xu, K. W. Cheng, and J. M. Guerrero, "A model predictive control strategy of pv-battery microgrid under variable power generations and load conditions," Applied Energy, vol. 221, pp. 195-203, 2018.

[17] Y. Shi, H. D. Tuan, A. V. Savkin, C.-T. Lin, J. G. Zhu, and H. V. Poor, "Distributed model predictive control for joint coordination of demand response and optimal power flow with renewables in smart grid," Applied Energy, vol. 290, Article ID 116701, 2021.

[18] D. Bertsimas and M. Sim, “The price of robustness," Operations Research, vol. 52, no. 1, pp. 35-53, 2004.

[19] W. Gu, Z. Wang, Z. Wu, Z. Luo, Y. Tang, and J. Wang, “An online optimal dispatch schedule for cchp microgrids based on model predictive control," IEEE Trans. Smart Grid, vol. 8, no. 5, pp. 2332-2342, 2016.

[20] A. J. Sguarezi Filho, M. E. de Oliveira Filho, and E. Ruppert Filho, "A predictive power control for wind energy," IEEE Trans. Sustainable Energy, vol. 2, no. 1, pp. 97-105, 2010.

[21] X. Serrano-Guerrero, M. Briceño-León, J.-M. Clairand, and G. Escrivá-Escrivá, "A new interval prediction methodology for short-term electric load forecasting based on pattern recognition," Applied Energy, vol. 297, Article ID 117173, 2021.

[22] Z. Wu, H. Zhan, W. Gu, S. Zheng, and B. Li, "Interval state estimation of distribution network with power flow constraint," IEEE Access, vol. 6, pp. 40826-40835, 2018.

[23] Y. Hua, J. Guo, and H. Zhao, "Deep belief networks and deep learning," in Proceedings of the 2015 International Conference 
on Intelligent Computing and Internet of Things, pp. 1-4, IEEE, Harbin, China, January 2015.

[24] I. Sutskever and G. E. Hinton, "Deep, narrow sigmoid belief networks are universal approximators," Neural Computation, vol. 20, no. 11, pp. 2629-2636, 2008. 